Mens

revue d'histoire intellectuelle de l'Amérique française

Ronald Rudin. Remembering and Forgetting in Acadie: $A$

Historian's Journey Through Public Memory, Toronto, University of Toronto Press, 2009, 350 p.

\title{
Joel Belliveau
}

Volume 11, numéro 2, printemps 2011

URI : https://id.erudit.org/iderudit/1023374ar

DOI : https://doi.org/10.7202/1023374ar

Aller au sommaire du numéro

Éditeur(s)

Centre de recherche en civilisation canadienne-française

ISSN

1492-8647 (imprimé)

1927-9299 (numérique)

Découvrir la revue

Citer ce compte rendu

Belliveau, J. (2011). Compte rendu de [Ronald Rudin. Remembering and

Forgetting in Acadie: A Historian's Journey Through Public Memory, Toronto,

University of Toronto Press, 2009, 350 p.l Mens, 11(2), 99-104.

https://doi.org/10.7202/1023374ar 


\section{Comptes rendus}

\section{Ronald Rudin. Remembering and Forgetting in Acadie: A Historian's Journey Through Public Memory, Toronto, University of Toronto Press, 2009, 350 p.}

Avec Remembering and Forgetting in Acadie, Ronald Rudin apporte une contribution originale à l'étude des commémorations. Ce spécialiste en histoire intellectuelle et culturelle du Canada francophone, bien connu pour ses travaux (et sa participation aux débats) sur l'historiographie québécoise, nourrit depuis quelques années un intérêt grandissant pour l'histoire publique. Ayant publié en 2003 un ouvrage sur ce thème en contexte québécois (Founding Fathers: Champlain and Laval in the Streets of Quebec), il a ensuite participé à la création du programme d'histoire publique à l'Université Concordia en 2005. Voici qu'il propose une deuxième monographie sur la même thématique, mais en déplaçant son regard, cette fois, vers l'Acadie et vers le présent.

Rudin dit avoir eu l'idée de ce livre alors qu'il travaillait sur le tricentenaire de Québec. En se penchant sur les commémorations passées, il s'est rendu compte que les historiens " have been limited by the chance survival of documentation». Il affirme à cet égard : "I frequently wished that I might have had the chance to talk to Earl Gray (the governor general who was the mastermind behind the tercentenary)" (p. 6). C'est à ce moment qu'il a conçu le projet de documenter la planification d'anniversaires importants de l'histoire des provinces maritimes, qui allaient avoir lieu en 2004 et 2005 , soit le $400^{\circ}$ anniversaire des premières tentatives de colonisation française en Amérique $(1604,1605)$ et le $250^{c}$ anniversaire de la Déportation des Acadiens (1755).

C'est dans ce contexte que Rudin a pris le chemin du NouveauBrunswick et de la Nouvelle-Écosse à plusieurs reprises pendant ces 
deux années afin de participer aux activités de commémoration en tant que "embedded historian" (terme emprunté aux forces militaires américaines de l'heure, dont les contingents en Irak ont leurs "embedded journalists»). Cette méthode - souvent employée par les sociologues et les anthropologues, qui l'appellent « observation participante " - a visiblement eu une influence importante sur cet historien. Ce n'est pas tous les jours que les historiens se frottent aux sensibilités subjectives de multiples individus en chair et en os, ou qu'ils sont forcés de reconnaître que les événements historiques appartiennent, en fin de compte, à tout le monde. Outre ses observations personnelles, Rudin se sert aussi de sources d'information plus classiques, amassant de grandes quantités de documents sur l'organisation des événements. Plusieurs de ces documents ont d'ailleurs été obtenus grâce à des demandes d'accès à l'information auprès du gouvernement fédéral (qui entretenait des liens avec la totalité des acteurs en présence). L'originalité du projet de Rudin par rapport aux autres études portant sur la commémoration repose donc sur le fait qu'il documente la mise sur pied de projets mémoriels contemporains, sur le vif et en temps réel.

Non seulement Rudin innove par sa manière d'aborder le sujet, mais il innove aussi en ce qui concerne la forme. Le livre ne représente en effet qu'un volet d'un projet plus vaste de documentation, d'étude et de discussion de ces commémorations. En parallèle, un film documentaire a été tourné (Life After Ille Ste-Croix, Office national du film, 2006) et un site Internet a été créé (Remembering Acadie, [En ligne], [http://rememberingacadie.concordia.ca/]). Alors que le film doit être considéré comme un produit distinct du livre, le site Internet, lui, est à la fois un produit autonome (qui peut être consulté par les internautes n'ayant pas l'ouvrage entre les mains) et un outil d'accompagnement du livre. Par un système astucieux et convivial de renvois au site Internet, l'auteur accorde au lecteur un accès facile à une quantité impressionnante de renseignements bien classés. Photos (anciennes et récentes), vidéos et entrevues viennent ainsi compléter la narration de l'historien. L'expérience, qui allie l'unité du livre avec 
la sensualité du multimédia, a beaucoup pour plaire. On ne peut s'empêcher de se demander si l'avenir du livre (qu'il soit en format papier ou électronique) ne ressemblera pas à ça.

Comme c'était déjà le cas avec Founding Fathers, Rudin espère que ce nouvel ouvrage jettera de la lumière sur l'acte commémoratif de façon générale. Il s'interroge ainsi sur le rôle et le poids des acteurs individuels dans l'organisation des cérémonies et se demande s'il y a quelque chose d'inévitable dans la forme que prennent ultimement ces manifestations. Celles-ci représenteraient-elles un simple " miroir » des rapports de force entre les communautés culturelles en contact? $\mathrm{Ou}$, au contraire, des surprises peuvent-elles en émerger?

L'ouvrage est divisé en deux parties. La première, intitulée «Stories of Beginnings", comporte quatre chapitres qui portent sur les commémorations marquant l'établissement de colonies permanentes à l'ÎleSainte-Croix (1604) et à Port-Royal (1605) par Pierre Dugua, sieur de Mons et Samuel de Champlain. Le premier chapitre s'intéresse aux commémorations passées (tenues en 1904, 1954-1955, 1979), alors que les trois chapitres subséquents traitent des perspectives de trois groupes bien différents sur les commémorations de 2004-2005. Ils abordent ainsi les vues des Acadiens, des populations anglophones locales qui sont maintenant " the stewards of the sites" et enfin des autochtones de la région. La deuxième partie, "Stories of Trauma", est plus courte et traite des commémorations de la Déportation des Acadiens (1755) en deux chapitres : un premier examine les commémorations passées $(1905,1955)$ alors que le second se penche sur les événements mémoriels de 2005. Ces derniers ont été, entre autres choses, l'occasion d'un débat animé sur la nécessité (ou non) d'obtenir des excuses officielles pour la Déportation, un dossier ayant eu de multiples rebondissements de la Louisiane à Londres en passant par le Québec, Ottawa et, bien sûr, l'Acadie de l'Atlantique.

En cours de route, l'auteur nous présente l'histoire de chacune des populations touchées par ces anniversaires historiques, ainsi que leur participation (ou leur non-participation, dans certains cas) à ceux-ci. On fait la connaissance des Acadiens, mais aussi celle des 
populations anglophones de Saint-Andrews (petite ville sise près de l'Île-Sainte-Croix) et d'Annapolis Royal (nom donné à Port-Royal après sa prise par la Grande-Bretagne en 1713) ainsi que des communautés autochtones des environs, soit les Passamaquoddy du sud-ouest du Nouveau-Brunswick et de la réserve micmaque de Bear River, à quelques minutes d'Annapolis Royal.

Bien qu'il soit original et intéressant, l'ouvrage comporte néanmoins certains défauts. Le style employé n'est pas particulièrement enlevant. Certains faits, contextes et idées sont martelés et rappelés sans cesse sans que cela ne soit nécessaire. On s'enfonce par moments trop profondément dans les détails de querelles, d'échanges épistolaires ou de problèmes logistiques liés aux actes commémoratifs dans de longs passages qui n'apportent pas grand-chose à notre compréhension des enjeux mémoriels en présence. Sur le plan de la méthode, il est frustrant de constater que les nombreuses citations tirées de lettres ou de discours en français sont données seulement dans la traduction de l'auteur. Par ailleurs, la pluralité de points de vue incorporés est à l'origine de quelques difficultés narratives. L'ouvrage n'a finalement qu'un mince fil conducteur puisqu'il prend en compte les perspectives mémorielles de cinq communautés différentes (sans parler des gouvernements) par rapport à deux anniversaires très différents. Ayant choisi de structurer son livre autour de ces anniversaires, Rudin se retrouve avec un texte qui, bien que cohérent sur le plan thématique, comporte quelques inconvénients en matière de chronologie. Le lecteur voyage de 1904 jusqu'au présent dans la première partie de l'ouvrage et doit retourner en 1905 et recommencer le trajet dans la seconde. Ainsi, plusieurs fois, des événements ayant eu lieu à une seule année d'intervalle et impliquant les mêmes individus et organisations sont-ils décrits dans des parties tout à fait différentes du livre. Conséquemment, l'auteur a dû ajouter de nombreux rappels et mises en contexte afin de restituer l'unité chronologique des événements.

Cela dit, la diversité des voix présentes constitue aussi la richesse et la contribution principale de ce livre. Non seulement le lecteur est exposé aux points de vue de personnes et de groupes multiples 
(incluant des sous-groupes dans chaque communauté), mais il en vient à ressentir tour à tour de l'affection et de l'antipathie envers chacun. Poussés par leurs perceptions de la justice et de la vérité historique, tous et chacun sombrent par moments dans le nombrilisme, l'étroitesse d'esprit et la petite politique. Au-delà de ça, on assiste aussi à quelques échanges culturels significatifs empreints de compréhension mutuelle, même si elle demeure limitée. Par le jeu de l'intersubjectivité, Rudin nous livre ici une histoire profondément humaine.

On le devinera, l'ouvrage représente l'étude la plus achevée - et de loin - sur les actes commémoratifs en contexte acadien et " maritimien ». Il existait certes déjà quelques bons articles sur les commémorations de la Déportation. Rudin en fait non seulement la synthèse, mais il y va d'ajouts, de rectifications et d'interprétations différentes, basées sur l'examen des sources. En ce qui concerne les commémorations des fondations de Sainte-Croix et de Port-Royal, le terrain était pratiquement vierge. Par ailleurs, Rudin resitue bien ces événements dans les écrits théoriques portant sur l'acte commémoratif, faisant notamment un usage fécond des travaux de Peter Novick.

Au-delà des nombreuses intrigues ponctuelles ayant marqué tous ces actes commémoratifs, une tendance forte se dessine : celle de la prise de parole des minorités en matière de mémoire publique. Tant les Acadiens que les autochtones étaient largement exclus des commémorations marquant les débuts de la colonisation, qui ont été organisées au début et au milieu du xx $x^{\mathrm{e}}$ siècle. La présence des premiers se limitait à un ou deux dignitaires, invités pour la forme et à la dernière minute. Quant aux seconds, ils étaient présents seulement par procuration, sous les traits d'acteurs déguisés reconstituant la scène de l'arrivée en Amérique de l'expédition du sieur de Mons et de Champlain. Par ailleurs, on insistait beaucoup à l'époque sur le fait que c'était le début de la colonisation " européenne " de la région (et pas spécifiquement française) et on passait sous silence l'existence de la communauté acadienne contemporaine, pourtant héritière directe des événements commémorés. On mesure bien, dès lors, 
comment le ton de toute commémoration est intrinsèquement politique. La suite de l'ouvrage illustre deux prises de parole bien distinctes. Il y a celle des Acadiens, entamée - de façon hésitante, torturée, mais néanmoins courageuse - autour du bicentenaire de la Déportation, puis parachevée subséquemment, par étapes, changeant de ton à mesure que leur niveau de confiance collective augmentait. Il y a ensuite celle des autochtones, beaucoup plus tardive, qui se déroule essentiellement autour $\mathrm{du} 400^{\mathrm{e}}$ anniversaire de la colonisation. Signe de l'ampleur des changements culturels actuellement en cours, ce sont les Passamaquoddy et les Micmacs qui ont obtenu les plus grands gains de cette dernière ronde d'actes commémoratifs.

Malgré le constat de cette nouvelle tendance, l'étude de Rudin ne sombre pas dans une perspective téléologique. Il affirme à maintes reprises que les tentatives d'influer sur la mémoire populaire, même quand elles sont menées par des élites influentes, peuvent échouer. Il mentionne d'ailleurs de nombreux exemples de ces échecs, le plus important étant celui des organismes acadiens dans leur tentative de proposer un nouveau "mythe fondateur " aux francophones des Maritimes en 2004, mythe qui mettait davantage l'accent sur les débuts de la colonisation et moins sur la Déportation.

Bref, Ronald Rudin apporte ici une contribution importante au domaine de l'histoire publique en Acadie ainsi qu'une contribution qui ne manque pas d'originalité au champ plus large des études commémoratives. Ses mérites lui ont d'ailleurs permis de remporter le Prix en histoire publique 2011 de la Société historique du Canada.

- Joel Belliveau Département d'histoire Université laurentienne 